\title{
The design service life of wide-body commercial aircraft research based on airlines data
}

\author{
Yue Wang ${ }^{1, a}$, Dan $\mathrm{Gao}^{1}$ and Jiangtao $\mathrm{Si}^{1}$ \\ ${ }^{1}$ Shanghai Aircraft Design and Research Institute, No. 5188, Jinke Road, Pudong New Area, Shanghai, China
}

\begin{abstract}
To reasonably define the design service life of Chinese double aisles wide-body commercial aircraft, the exact market region and design condition are researched. The base market of Chinese double aisles wide-body commercial aircraft is China, Asia, Europe and the Pacific. In these zones, customers should be analyzed carefully, and the customers who are not available but play an important role in these zones should be picked off decisively. Besides, the flight length definition is also studied, which affects the design service life of aircraft obviously in small market region. After research, the results could reduce engineering difficulties, and also increase airlines adaptability and competitiveness in the specific market zone.
\end{abstract}

\section{Introduction}

Commercial aircraft is a special commodity, whose internal attribute asks it to meet the market requirements, which turns to be an important condition for success. Market is the source of product, so is in the aviation industry.

Design service life is a limited period confirmed during aircraft certification, displaying in the form of flight cycles and flight hours [1]. Design service life is an important fatigue evaluation parameter affecting the aircraft structure weight directly. So a reasonable design service life is critical to the market competitiveness and economy of the aircraft.

Currently, the method of minimum design service life is commonly used by most of aircraft manufacturers. Three typical flight lengths are provided with the method, including the short, medium and long characteristic flight length [2]. These flight lengths are calculated in general from the airlines data of market. Airlines are scattered all around the world, with different geographical location, natural environment, cities distribution, population distribution, geopolitical position, customers habits, etc., which leads to the unique regional features of different airline markets. So the aircraft manufacturers need to be sensitive to the market and produce the appropriate product to meet exactly requirement of the market.

\section{Analysis process of airlines data}

To analyze the airlines data effectively and find out the airlines feature reasonably, a bank of airlines data should be established firstly, then the constraints should be defined exactly, and finally the data

\footnotetext{
${ }^{a}$ Corresponding author : wangyue@comac.cc
} 
of object market could be picked out as a special data collection, which is the basis of analysis and research. The analysis process is shown in figure 1 as below.

In the whole process, the most important step is constraints definition, which defines the object market of product development [3] For indicating the design service life of double aisles wide body commercial aircraft clearly, three constraints are defined and applied in operation route analysis. The first one is the aircraft types, which classifies the aircraft by flight range and seats. With this constraint, a global object market data for special competitors is collected. The second one is geographical division, which reflects the detailed geographical region of object market. The third one is the customers, who will really operate the aircraft in the future. These constraints make preparation for the analysis to the exact object market data.

Following the process, the analysis results with different constraints would be shown in the paper.

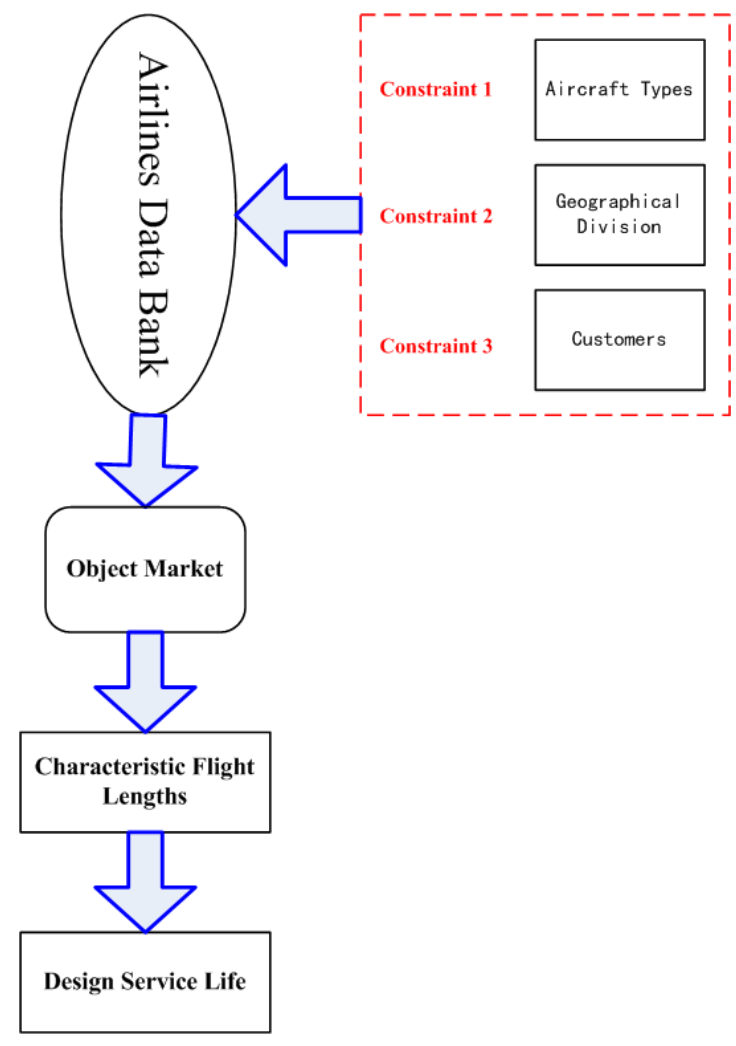

Figure 1. Analysis process of airlines data

\section{The method of aircraft design service life}

Aircraft design service life is a predefined life in aircraft design, which is achieved by design, maintenance and so on [4]. Generally the method of minimum design service life is used by most aircraft manufacturers, by which the design service life of aircraft could be analyzed rapidly and effectively. In the paper, this method would be used in the analysis.

Generally speaking, minimum design service life is the aircraft's serving life, 20 years at least, cumulated under the harshest operating conditions. During this period, there shall not be obvious cracks in main load-bearing structures of aircraft [2]. With this method, three special flight lengths are provided for defined the flight cycles, see figure 2 for details. 


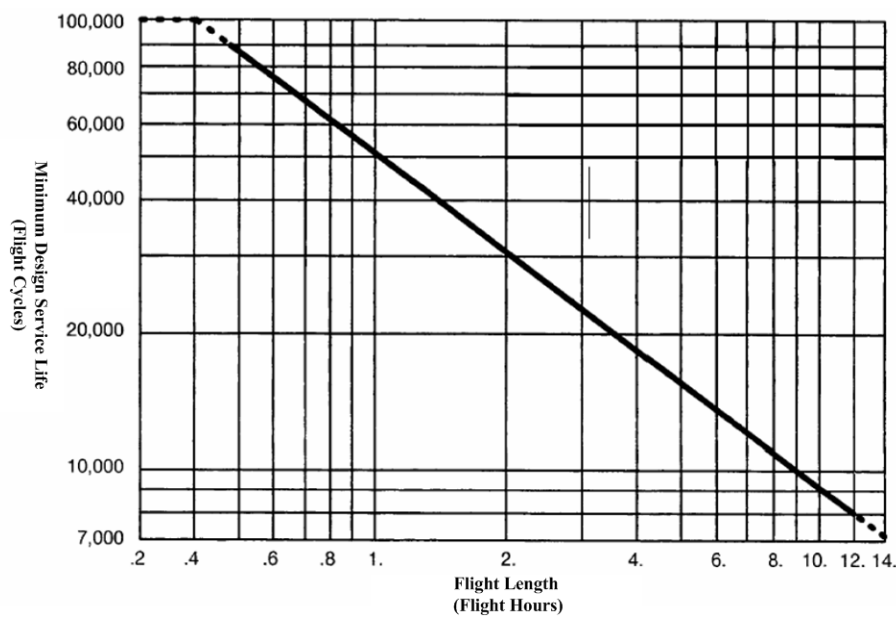

Figure 2. Minimum design service life curve [2]

With the development of commercial airlines, minimum design service life curve would be modified a little. In the paper, the fitting curve equation is approximate to

$$
M D S G=10^{(4.7-0.741 \lg h)}
$$

Where $\mathrm{h}$ is flight hour of a flight length [2].

\section{The airlines data analysis of wide-body aircrafts}

According to the analysis process of airlines data, the characteristics of different markets will be analyzed and shown.

\subsection{Global airlines data analysis of wide-body aircrafts}

In general, there are double aisles with 200 to 400 seats in commercial wide-body aircrafts, whose range is no less than $10000 \mathrm{~km}$, such as A330, A350, B787 and B777. But a special aircraft type of B767 should not be neglected, which has been excluded from the market. The airlines operated by B767 aircrafts have been replaced by other wide-body aircrafts gradually. So these airlines should be accounted in the global airlines data of wide-body aircrafts [5].

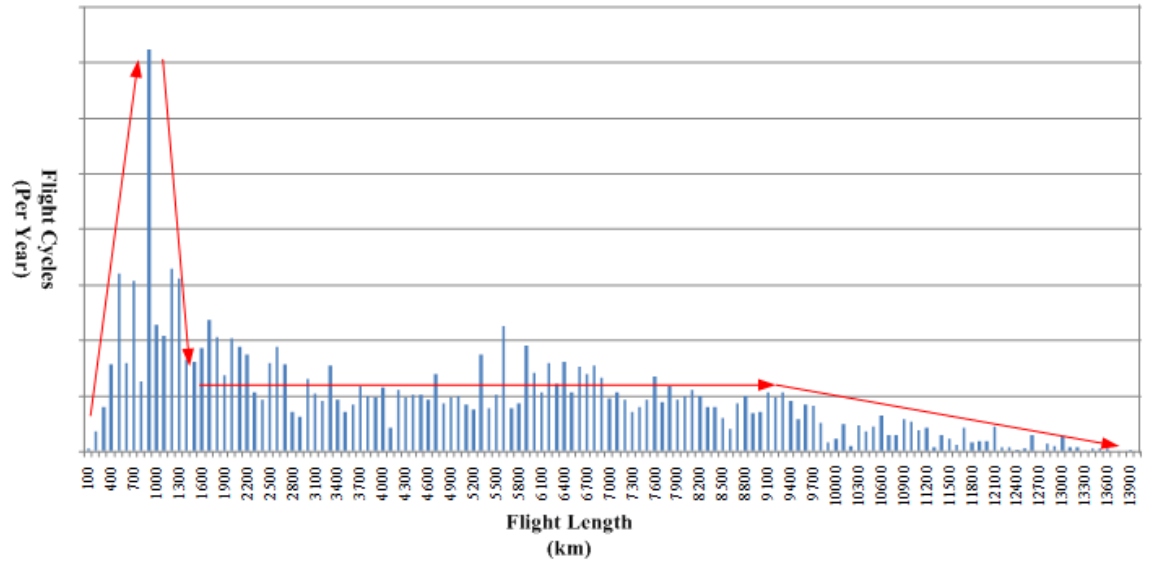

Figure 3. Global airlines distribution of wide-body aircrafts 
Figure 3 shows that there is an operating peak value at about $900 \mathrm{~km}$ flight length, which is much more than other flight lengths. This phenomenon reflects an explosive market in this flight length, which will be focused and analyzed in chapter 3.3. After the peak value till about $9000 \mathrm{~km}$ flight length, the operating frequencies become smooth. And after $9000 \mathrm{~km}$ flight length, they reduce gradually.

\subsection{Airlines data analysis of wide-body aircrafts in Asia-Europe-Pacific region}

The commercial aircrafts of China developed later, so the development goal is different from the western aircraft companies, who face the global market. For developing commercial wide-body aircraft of China, an appropriate and exact market should be focused and chosen, where the product would be competitive and get success easier.

There is a large advantage for commercial aircraft development of China, which is the huge inner market. According to the prediction, during 2025 to 2045, about 1300 new wide-body aircrafts are needed, which is about $13 \%$ of global new increasing wide-body aircrafts, so the inner market is big enough for the primary market of commercial wide-body aircrafts development of China. Otherwise, considering the belt and road strategy, the market of commercial wide-body aircrafts development of China is located in the region of Asia-Europe-Pacific.

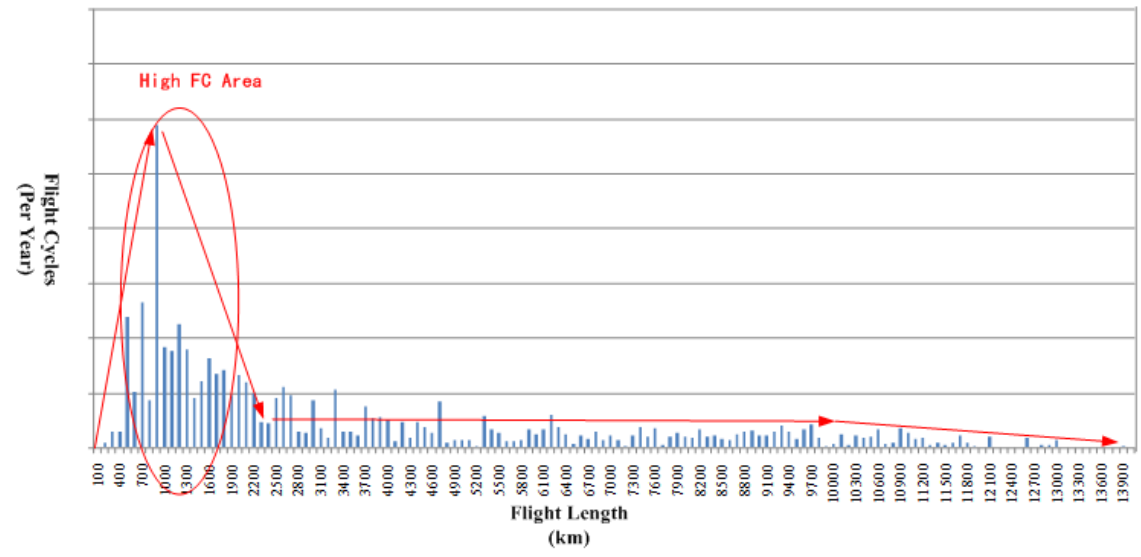

Figure 4. Asia-Europe-Pacific airlines of wide-body aircrafts distribution

Figure 4 is the similar as figure 3 in airlines distribution. In short flight length, there is a peak value at about $900 \mathrm{~km}$. The medium flight length frequencies are smooth. The long flight length frequencies are less than others.

There are large quantities of airlines operated with wide-body aircrafts in Asia-Europe-Pacific region. Because of the large population distribution and rapid economy development in these regions, this market will become more and more important.

For reaching the requirements of this important market, the potential customers should be analyzed carefully, and the interference information should be eliminated.

\subsection{Customers airlines data analysis of wide-body aircrafts in Asia-Europe-Pacific region}

Through analyzing and classifying the potential customers in object region, especially considering the wide-body aircrafts flight quantities per year, the market of Japan should be concentrated intensively. Because of its population and cities distribution, there are large quantities short-thick airlines operated by wide-body aircrafts in this market. Therefore, Boeing provides a special short range wide-body B787 aircraft according to the characteristics in Japanese airlines market. [4] 


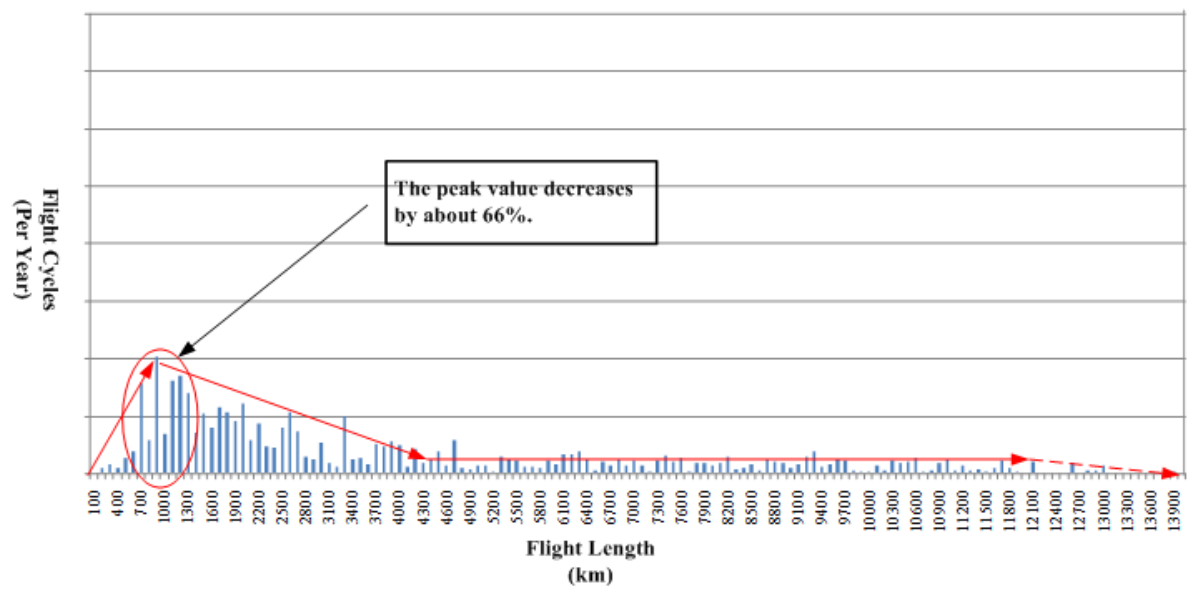

Figure 5. Customers airlines of wide-body aircrafts in AEP region distribution

There is a big different between figure 5 and figure 4. In figure 5 , the peak value of flight frequencies decreases by about $66 \%$, which comes from Japan airlines market of wide-body aircrafts. Considering the conventional operating habits of Japan and the difficulties in products design, eliminating the Japanese market from the customers airlines of Chinese wide-body aircrafts in AEP region is proposed.

\subsection{The characteristic flight lengths for design service life}

Based these markets mentioned above, the characteristic flight lengths have been calculated with a fixed proportion of flight frequencies.

Table 1. The characteristic flight lengths in different object markets of wide-body aircrafts

\begin{tabular}{|c|c|c|c|}
\hline Market Region & $\begin{array}{c}\text { Short length } \\
\text { km }\end{array}$ & $\begin{array}{c}\text { Medium length } \\
\text { km }\end{array}$ & $\begin{array}{c}\text { Long length } \\
\text { km }\end{array}$ \\
\hline Globe & 1290 & 5850 & 10425 \\
\hline Asia-Europe-Pacific(AEP) & 880 & 3830 & 10165 \\
\hline Customers in AEP & 1165 & 4400 & 10510 \\
\hline
\end{tabular}

Table 1 shows that the characteristic flight lengths are different in different object markets, especially in short lengths and medium lengths. Primarily because the special market of wide-body aircrafts of Japan exists. For long lengths, they are almost the same, which reflects the long flight length airlines distribute evenly globally.

\section{The design service lives of wide-body aircrafts in different object markets}

Table 2. The design service lives of wide-body aircrafts in different object markets

\begin{tabular}{|c|c|c|}
\hline Market Region & Flight cycles & Flight hours \\
\hline Globe & 32000 & 100000 \\
\hline Asia-Europe-Pacific(AEP) & 42000 & 100000 \\
\hline Customers in AEP & 34000 & 100000 \\
\hline
\end{tabular}

In design service life calculation of wide-body aircraft, the short length basically reflects the flight cycles. In table 1 , the short length of Asia-Europe-Pacific increases by about $32.3 \%$, from $880 \mathrm{~km}$ to $1164 \mathrm{~km}$, after eliminating Japan and Korea market data. And in table 2, the flight cycles decrease 
about $19 \%$. This is a significant result, which means the more appropriately the market is chosen, the more competitive the product would be.

\section{Flight length divisions effecting on the design service life of wide- body aircraft}

In above analysis, the characteristic flight lengths for design service life are defined with a fixed proportion of flight frequencies, which means separately fixed percentages of flight times indicating the short, medium and long flight length. This definition method shows advantages in calculation stability in large sample space. But in small sample space, because of discontinuity of data, the calculation result turns out to be not precise enough.

To analyze the special market exactly, it is necessary to redefine the short, medium and long flight lengths. There are two elements related directly with the commercial aircrafts, which are the airline and the passenger, so these two elements are considered primarily in this paper.

At first, short flight length is redefined. For airline companies, the airlines operated with widebody aircrafts are almost short-thick airlines, which carry large quantities of passengers. When conditions are matched, it is easy to initiate the airline. So for the short flight length definition, more attention should be paid to the requirements of passengers. For business passengers attending business meetings in different cities would be hoped in one day. And for short distance tourists, a shorter time spent in route the better, just like self-driving travel in weekend. Through a limited sample inquiry, the result is that the short flight length is no more than 3 flight hours. This inquiry will be researched in detail in the future.

Long flight length definition is opposite with short flight length definition. The focus of the former is the aircraft operator. For spending long time in air, the pilot weary of flight has the possibility to make mistakes. So the flight hours of pilot is restricted to 10 hours in both CCAR91 and CCAR21.

Therefore, the medium flight length is redefined between 3 to 10 flight hours.

According to the new flight lengths divisions, the characteristic flight lengths are re-analyzed.

Table 3. The characteristic flight lengths of wide-body aircrafts based on new flight lengths definition

\begin{tabular}{|c|c|c|c|}
\hline Market Region & $\begin{array}{c}\text { Short length } \\
\text { km }\end{array}$ & $\begin{array}{c}\text { Medium length } \\
\mathbf{~ k m}\end{array}$ & $\begin{array}{c}\text { Long Length } \\
\mathbf{~ k m}\end{array}$ \\
\hline Globe & 1225 & 5470 & 10225 \\
\hline Asia-Europe-Pacific(AEP) & 1215 & 4830 & 10325 \\
\hline Customers in AEP & 1355 & 4765 & 10450 \\
\hline
\end{tabular}

Three characteristic flight lengths in Table 3 are almost the same with Table 1 in global market region, which reflects the new flight lengths definition is reliable. But more attention should be paid to the smaller market region, the result is different obviously, especially in short flight length.

Table 4. The design service life of wide-body aircrafts based on new flight lengths definition

\begin{tabular}{|c|c|c|}
\hline Market region & Flight Cycles & Flight Hours \\
\hline Globe & 32000 & 100000 \\
\hline Asia-Europe-Pacific(AEP) & 32000 & 100000 \\
\hline Customers in AEP & 30500 & 100000 \\
\hline
\end{tabular}

In Table 4, the design service lives of wide-body aircrafts are different in smaller market region. In the customers' market region, the design service life could decrease by $10.3 \%$, which is a significant benefit for aircraft design. Therefore, to increase the competitiveness in the special market of widebody aircrafts, the exact design condition should be researched and defined. 


\section{Conclusions}

The airlines data of wide-body aircrafts has been analyzed carefully, and the following results have been achieved:

a). The airlines distribution characteristics in different market regions are different.

b). Japanese wide-body airlines market operating wide-body aircrafts is a special market, because of its population and cities distribution, there are large quantities short-thick airlines operated by wide-body aircrafts in this market. In the flight frequencies distribution chart, this market could make a peak value in a short length region, which affects design service life of wide-body aircrafts a lot.

c). The flight length division should be researched. In analysis of airlines data in special market regions, this effect element plays an important role to increase the competitiveness.

d). The base market of Chinese double aisles wide-body commercial aircraft is China, Asia and the Pacific. In this zone, customers should be analyzed carefully, and the customers who are not available but play an important role in this zone should be picked off decisively. The exact market definition for Chinese double aisles wide-body commercial aircraft could reduce engineering difficulties, and also increase airlines adaptability and competition in the specific market zone.

\section{References}

1. CAAC. CCAR PART 25, AIRWORTHINESS STANDARDS: TRANSPORT CATEGORY AIRPLANES[S]. 2011.11. (in Chinese)

2. The damage-tolerance and durability of aircraft structure design manual, volume 2, the fatigue analysis of aircraft structure[M]. Beijing: Aviation Industry Press, 1989. (in Chinese)

3. Chen Bushi, Li Yunjun, Wang Zhiyu. Aircraft Design Manual, volume 5, Civil Aircraft General Design[M]. Beijing: Aviation Industry Press, 2005.8, 16 34. (in Chinese)

4. Egbert Torenbeek. Synthesis of subsonic airplane design[M]. Netherlands: Delft University Press, 1976.

5. The market research center of COMAC. The market prediction annual report of COMAC(20162035)[R]. 2016.11. (in Chinese) 